\title{
Dynamics of resonant optical waveguide semiconductor laser arrays
}

\author{
Sujatha Ramanujan and Herbert G. Winful \\ Department of Electrical Engineering and Computer Science and Center for Ultrafast Optical Science, \\ University of Michigan, University of Michigan, 1301 Beal Avenue, Ann Arbor, Michigan 48109
}

(Received 12 November 1992; accepted for publication 31 March 1993)

\begin{abstract}
A propagation model is presented for the dynamics of antiguided semiconductor laser arrays. The model takes into account diffraction, carrier diffusion, spatial hole burning, and carrier-induced antiguiding. Numerical results reveal a variety of spatiotemporal behaviors ranging from stable, quiescent operation to periodic and erratic intensity oscillations.
\end{abstract}

Phased locked arrays of antiguided semiconductor lasers have recently been developed in response to the need for compact sources that emit high power into a single diffraction limited beam. ${ }^{1,2}$ Much effort has gone into understanding the spatial properties of these arrays and optimizing their output power and mode discrimination qualities. To date however, there have been no published studies of the temporal dynamics of these lasers. In this letter we present the results of a theoretical investigation of the dynamics of antiguided semiconductor laser arrays. The analysis is based on a propagation model we have previously used to describe the dynamics of gain-guided semiconductor laser arrays. ${ }^{3}$ Our results show that the temporal stability of these antiguided arrays is highly dependent on the pump current level. Gain saturation and carrier-induced antiguiding can lead to sustained temporal oscillations in the output power as well as asymmetries in the beam profile. In addition, we find saturable-absorber induced self-pulsation under certain conditions.

An antiguiding array consists of a number of coupled leaky waveguides (low index regions separated by high index regions). Coupling between the antiguides is by means of interference between the radiation fields of the leaky modes. In a resonant optical waveguide (ROW) array, the spacing between the antiguides is an integral number of half-wavelengths in the lateral direction. ${ }^{4}$ The resonant mode is termed "in-phase" or "out-of-phase" if there are, respectively, an odd or even number of half wavelengths in the interelement region.

To study the spatiotemporal evolution of the field in the array, we use the propagation model. ${ }^{3}$ The variation of the complex permittivity in the lateral direction is described by;

$$
\begin{aligned}
\Delta \epsilon= & 2 n_{a} \Delta n_{\mathrm{eff}}-\frac{a n_{a} \alpha\left(N-N_{0}\right)}{k_{0}}-i \frac{n_{a}}{k_{0}} a\left(N-N_{0}\right) \\
& +i \frac{n_{c}}{k_{0}} \alpha_{c} \frac{(1-\Gamma)}{\Gamma} .
\end{aligned}
$$

In Eq. (1) $n_{a}$ is the active region index, $\Delta n_{\mathrm{eff}}$ is the index step, $a$ is the linear gain, $\alpha$ is the linewidth enhancement factor, $N$ is the carrier density, $k_{0}$ is the free-space propagation constant, $N_{0}$ is the carrier density required for transparency, $n_{c}$ is the cladding index, $\alpha_{c}$ is the cladding loss, and $\Gamma$ is the confinement factor. Typical values of the parameters in Eq. (1) are given in Table I., The first term in Eq. (1) is the real index step. For the ROW devices this value is carefully selected to meet the criterion for mode discrimination. The second term includes the effect of carrier induced antiguiding on the value of the index of refraction. The linewidth enhancement factor $\alpha$ multiplies the carrier density and the product is linearly proportional to the change in real index. This term is strongly dependent on the pump level and can significantly alter the built-in index step. Consequently, the index step is a dynamic quantity. The third term is the gain and the final term is material loss. The field is propagated back and forth through the cavity as described by ${ }^{3}$

$$
\pm 2 i k \frac{\partial \Psi}{\partial z}+\frac{\partial^{2} \Psi}{\partial x^{2}}+k_{0}^{2} \Gamma \Delta \epsilon(r) \Psi_{ \pm}(x, z)=0,
$$

where $x$ is the lateral direction, $z$ is the propagation, $c$ is the speed of light, $n_{e}$ is the effective index, and $\Psi$ is the wave function. At the end of every lengthwise traversion the field is multiplied by the end mirror loss. Coupled to the wave equation is the carrier diffusion Eq. (3) given as

$$
\frac{\partial N}{\partial t}=p(x)-\frac{N(x, t)}{\tau_{s}}=g^{\prime}\left(N-N_{0}\right)|\Psi(x, t)|^{2}+D \frac{\partial^{2} N}{\partial x^{2}},
$$

\begin{tabular}{|c|c|c|c|}
\hline Parameter & Symbol & Value (Twin) & Value (10 elem.) \\
\hline Wavelength & $\lambda$ & $0.86 \mu \mathrm{m}$ & $0.86 \mu \mathrm{m}$ \\
\hline Cavity length & $L$ & $250 \mu \mathrm{m}$ & $750 \mu \mathrm{m}$ \\
\hline Stripe width & $d$ & $3 \mu \mathrm{m}$ & $4 \mu \mathrm{m}$ \\
\hline $\begin{array}{l}\text { Interelement } \\
\text { spacing }\end{array}$ & $s$ & $1 \mu \mathrm{m}$ & $2 \mu \mathrm{m}$ \\
\hline Index step & $\Delta n$ & $4 \times 10^{-2}$ & $4 \times 10^{-2}$ \\
\hline $\begin{array}{l}\text { Confinement } \\
\text { factor }\end{array}$ & $\Gamma$ & 0.2 & 0.2 \\
\hline $\begin{array}{l}\text { Active region } \\
\text { index }\end{array}$ & $n_{a}$ & 3.59 & 3.59 \\
\hline Cladding index & $n_{c}$ & 3.35 & 3.35 \\
\hline Gain & $a$ & $1.5 \times 10^{-8} \mu \mathrm{m}$ & $1.5 \times 10^{-8} \mu \mathrm{m}^{3}$ \\
\hline Antiguiding & $\alpha$ & 2.0 & 2.0 \\
\hline $\begin{array}{l}\text { Passive } \\
\text { absorption }\end{array}$ & $\alpha_{c}$ & $10 \mathrm{~cm}^{-1}$ & $10 \mathrm{~cm}^{-1}$ \\
\hline Carrier lifetime & $\tau_{S}$ & $2 \mathrm{~ns}$ & $2 \mathrm{~ns}$ \\
\hline $\begin{array}{l}\text { Diffusion } \\
\text { constant }\end{array}$ & $\ddot{D}$ & $\begin{array}{c}4.5 \times 10^{-3} \mu \mathrm{m}^{2} / \mathrm{ps} \\
\left(L_{p}=3 \mu \mathrm{m}\right)\end{array}$ & $\begin{array}{c}12.5 \times 10^{-3} \mu \mathrm{m}^{2} / \mathrm{ps} \\
\left(L_{p}=5 \mu \mathrm{m}\right)\end{array}$ \\
\hline Reffectivity & $R$ & 0.3 & 0.3 \\
\hline $\begin{array}{l}\text { Carrier Density } \\
\text { for transparency }\end{array}$ & $N_{0}$ & $1.2 \times 10^{6} \mu \mathrm{m}^{--3}$ & $1.2 \times 10^{6} \mu \mathrm{m}^{-3}$ \\
\hline $\begin{array}{l}\text { Interelement } \\
\text { loss }\end{array}$ & $\alpha_{i}$ & $50.0 \mathrm{~cm}^{-1}$ & $50.0 \mathrm{~cm}^{-1}$ \\
\hline
\end{tabular}

TABLE I. Device parameters. 


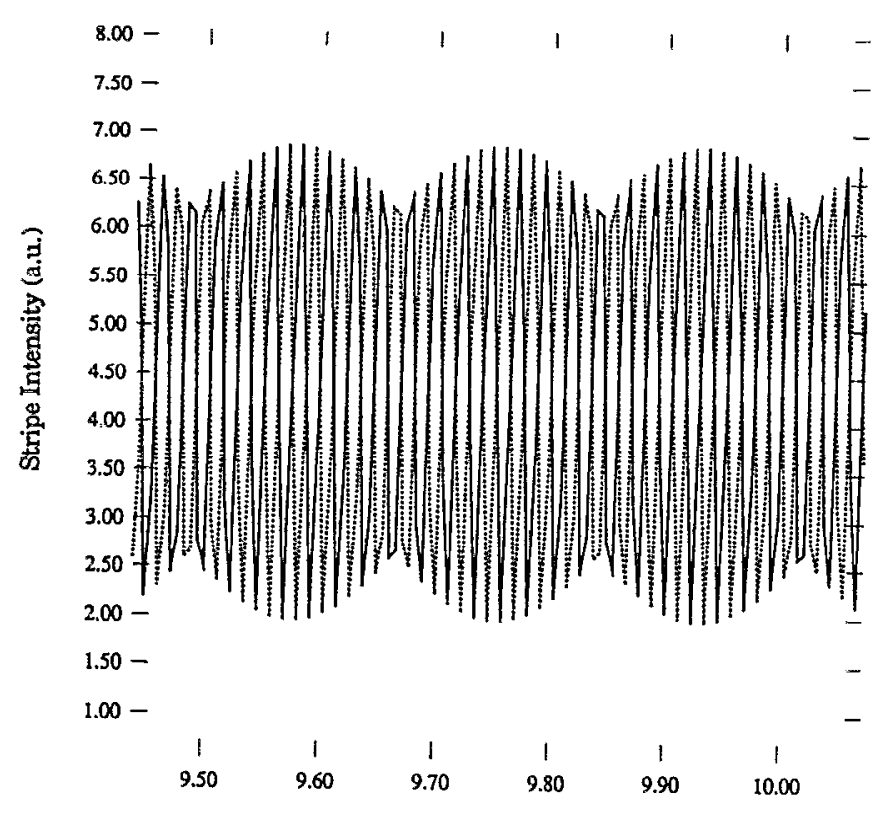

Time in ns

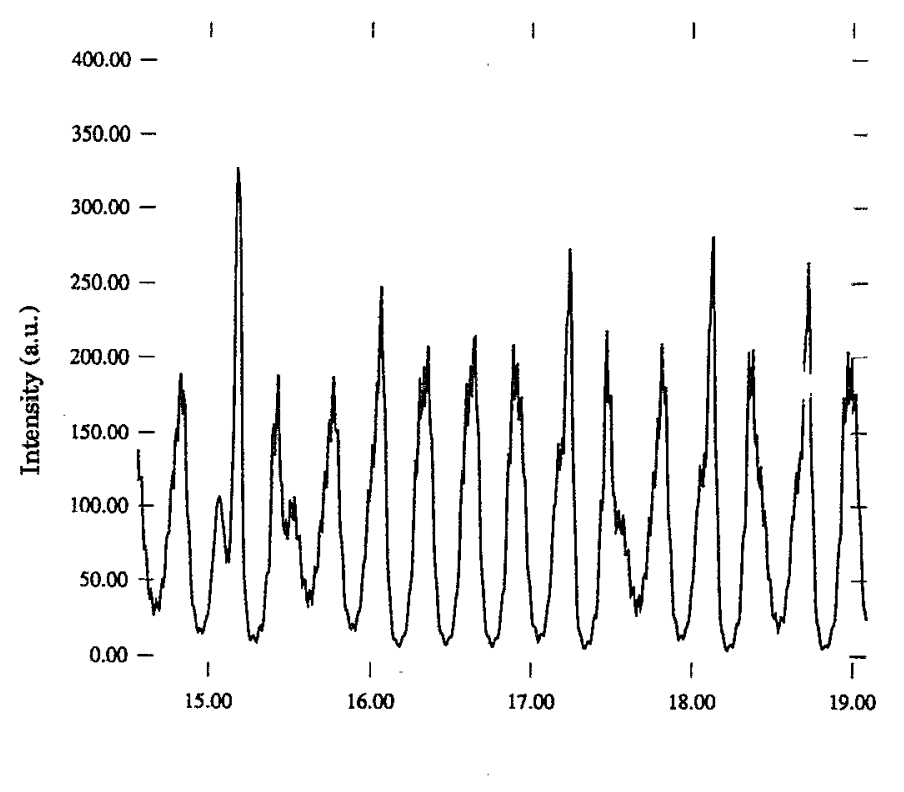

Time in ns

FIG. 2. Total intensity vs time for ten element temporally unstable array.

FIG. 1. Stripe intensity vs time for twin element temporally unstable array.

with the variables as defined in Table I. For a given pump current $p(x)$ given in carriers per cubic micron per picosecond, a carrier lifetime $\tau_{s}$, diffusion constant $D$, and differential gain $g^{\prime}=\Gamma c n_{a} a /\left(n_{c} n_{e}\right)$, Eqs. (1), (2), and (3) are solved using the Crank-Nicolson method with transparent boundary conditions. ${ }^{7}$

To understand the dynamics of leaky wave coupling, we first simulate a twin element ROW. The parameters are given in the third column of Table I. For pump currents less than $70 \mathrm{~mA}$ lasing occurs in the high index regions. These are the usual modes of evanescent-coupled index guided lasers. When the current is raised to $120 \mathrm{~mA}$, there is stable, out-of-phase lasing in the low index region with saturated gain. In the near-field, the lasing peaks are shifted towards the high index region which reflects a compromise between the passive mode of the structure, the index guide mode confined in the high index region, and the out of phase mode of the Fabry-Perot structure. The resulting lasing peak is not centered within the guide. At twice threshold ( 10000 carriers $/ \mu \mathrm{m}^{3} / \mathrm{ps}$ ), the laser output is unstable. The temporal profile shows a swapping of power as well as an overall envelope as can be seen in Fig. 1. The swapping of power can be explained as the beating of two symmetric modes. These modes arise from the superposition of the passive mode and the symmetric out-ofphase modes. The gain saturates symmetrically about the center of each guide, but the lasing mode is shifted towards the high index region. The result is the growth of the other symmetric mode in the region of excess gain. These two modes oscillate in power and produce the swapping behavior shown in Fig. 1. The overall envelope is the result of the pulling of the field into the region of high index. This pull- ing can be so strong that when the laser is unstable and mode swapping occurs, the lasing peak exits the guide region periodically.

Perhaps one of the strongest effects that corrupt the temporal stability of the device is the antiguiding factor. Carrier antiguiding enhances mode competition so strongly, it causes instabilities to arise at twice threshold or at threshold when otherwise the device is stable up to 4 time threshold. Because the index step has been altered through the pump current [the second term in Eq. (1)], the lateral traveling wave no longer sees an integral number of wavelengths and does not reach a null at the index interface. Thus, there is no longer a single lateral mode that defines the device operation.

Having explained the twin elements dynamics, we analyze the behavior of the ten element devices. For the set of parameters shown in the fourth column of Table I, we find no temporally stable solutions. In addition to the previously mentioned mode swapping instability, the array exhibits regions of self-pulsing as seen in Fig. 2. At 2.5 times threshold, the array displays a transient mode swapping instability before pulsing occurs. The output intensity pulsates at a few gigahertz frequency with all the elements acting in unison. The origin of the pulsations is the absorbing regions between the pumped antiguiding regions. The absorption can be saturated if there are significant intensity peaks in the interelement regions. Saturation of absorption results in repetitive $Q$-switching, a phenomena seen also in gain-guided arrays. ${ }^{9}$ With the larger arrays, there are a sufficient number of interelement regions to act as saturable absorbers so the array more readily instantaneously pulses. Figure 3 shows near-field profiles taken at a peak (solid line) and valley (dotted line) of the pulse train. Note the substantial interelement field and the asymmetry in the lateral profile. This asymmetry may be related to the 


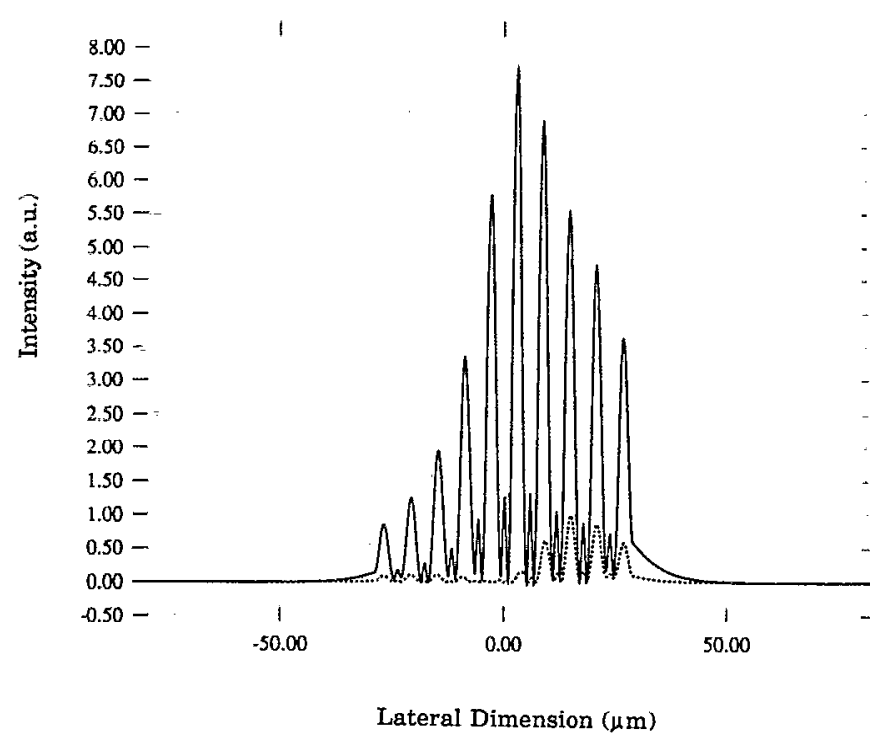

FIG. 3. Near-field intensity for a ten element laser. Solid line is the intensity at the peak of the pulse, dashed line is the intensity at the bottom of the pulse

lateral mode instability described by Land and others. ${ }^{10}$ Additionally, we observe spatial hole burning in the guide at the top of the pulse. In the far-field we also observe intensity pulsations and exchange of energy between the lobes of the field distribution. Figure 4 shows the far-field profile at two instants in time separated by $3 \mathrm{~ns}$. We remark that the temporal behavior of the 10 element array is quite complicated. In addition, to the strong self-pulsation described above, there is also the higher frequency mode competition described earlier that manifests itself as a ripple on top of the pulses.

In conclusion, we note that the simple design of the ROW laser exhibits multimode and hence unstable behavior at pump currents as low as twice threshold. These instabilities are not readily detectable without the use of high speed detectors and the ability to resolve the individual stripe intensities. The multimode behavior is greatly aggravated by the antiguiding effect. Through the antiguiding effect, the pump current alters the index of refraction sufficiently to deviate from the resonance condition require for ROW operation. Reduction of the antiguiding factor

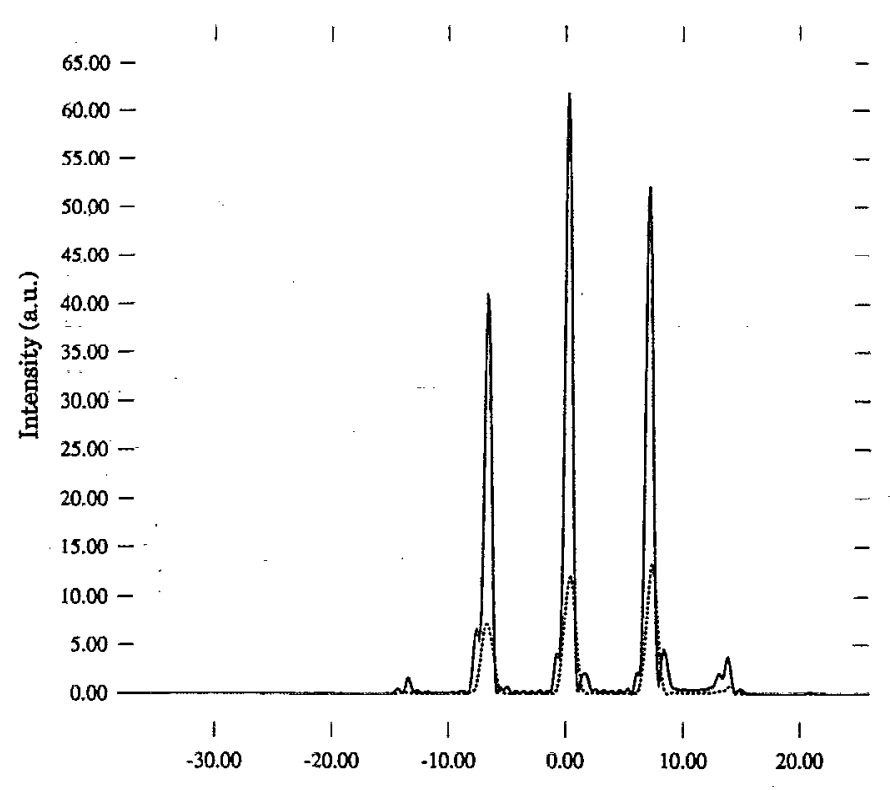

Lateral Dimension (a.u.)

FIG. 4. Far-field intensity patterns taken 3 ns apart for ten stripe array.

and diffusion provides a greater region of stability and encourages single mode behavior.

This research is supported in part by the National Science Foundation under Grant ECS-9806214 and through the Center for Ultrafast Optical Science under Grant STC PHY 8920108. Additional funding from the AFOSR is gratefully acknowledged.

${ }^{1}$ D. Botez, L. Mawst, G. Peterson, and T. Roth, Appl. Phys. Lett. 54, 2183 (1989).

${ }^{2}$ G. Hadley, D. Botez, and L. Mawst, IEEE J. Quantum Electron. 27, 921 (1991).

${ }^{3}$ S. S. Wang and H. G. Winful, J. Appl. Phys. 73, 462 (1993).

${ }^{4}$ D. Botez, L. Mawst, G. Peterson, and T. Roth, IEEE J. Quantum Electron. 26, 482 (1990).

${ }^{5}$ G. P. Agrawal, J. Appl. Phys. 56, 3100 (1984).

${ }^{6}$ L. J. Mawst, D. Botez, C. Zmudzinski, and C. Tu, Appl. Phys. Lett. 61, 503 (1992).

${ }^{7}$ G. Hadley, Opt. Lett. 16, 624 (1991).

${ }^{8}$ D. Botez, IEE Proc. J. 139, 14 (1992).

${ }^{9}$ B. S. Poh and T. E. Rozzi, IEEE J. Quantum Electron. 17, 723 (1981).

${ }^{10}$ R. Lang, IEEE J. Quantum Electron. 15, 718 (1979). 